\title{
New Jacobi Elliptic Function Solutions for the Kudryashov-Sinelshchikov Equation Using Improved F-Expansion Method
}

\author{
Yinghui He \\ Department of Mathematics, Honghe University, Mengzi, Yunnan 661100, China \\ Correspondence should be addressed to Yinghui He; heyinghui07@163.com
}

Received 3 May 2013; Revised 3 July 2013; Accepted 3 July 2013

Academic Editor: Gradimir Milovanovic

Copyright (C) 2013 Yinghui He. This is an open access article distributed under the Creative Commons Attribution License, which permits unrestricted use, distribution, and reproduction in any medium, provided the original work is properly cited.

Based on the F-expansion method with a new subequation, an improved F-expansion method is introduced. As illustrative examples, some new exact solutions expressed by the Jacobi elliptic function of the Kudryashov-Sinelshchikov equation are obtained. When the modulus $m$ of the Jacobi elliptic function is driven to the limits 1 and 0 , some exact solutions expressed by hyperbolic function and trigonometric function can also be obtained. The method is straightforward and concise and is promising and powerful for other nonlinear evolution equations in mathematical physics.

\section{Introduction}

It has recently become more interesting to obtain exact solutions of nonlinear partial differential equations. These equations are mathematical models of complex physical phenomena that arise in engineering, applied mathematics, chemistry, biology, mechanics, physics, and so forth. Thus, the investigation of the traveling wave solutions to nonlinear evolution equations (NLEEs) plays an important role in mathematical physics. A lot of physical models have supported a wide variety of solitary wave solutions.

In 2010, Kudryashov and Sinelshchikov [1] introduced the following equation:

$$
\begin{aligned}
& u_{t}+\gamma u u_{x}+u_{x x x} \\
& \quad-\varepsilon\left(u u_{x x}\right)_{x}-\kappa u_{x} u_{x x}-v u_{x x}-\delta\left(u u_{x}\right)_{x}=0,
\end{aligned}
$$

where $\gamma, \varepsilon, \kappa, \nu$, and $\delta$ are real parameters. Equation (1) describes the pressure waves in the liquid with gas bubbles taking into account the heat transfer and viscosity. When $\varepsilon=$ $\kappa=\delta=0$ and $\varepsilon=\kappa=\nu=\delta=0$, (1) becomes the BKdV equation and the KdV equation, respectively. So, (1) can be considered as the generalization of KdV equation. Therefore, the study to (1) is more meaningful than $\mathrm{KdV}$ equation and BKdV equation. We call this equation the KudryashovSinelshchikov equation.

Equation (1) was studied by many researchers in various methods. In the case of $v=\delta=0$, it was studied by Ryabov, using a modification of the truncated expansion method [2], by Randrüüt in a more straightforward manner [3], by Li et al., using the bifurcation method of dynamical systems [4-6], by Nadjafikhah and Shirvani-Sh, using the Lie symmetry method [7], and by He, using $G^{\prime} / G$-expansion method [8]. In the case of $\nu \neq 0, \delta \neq 0$, (1) was studied by Efimova using the modified simplest equation method [9], by Mirzazadeh and Eslami, using first integral method [10]. And they obtained some results when $\beta$ took special values.

We noticed that the Jacobi elliptic function solutions of (1) are only reported in [8] with special case $\beta=-3$ and $\beta=$ -4 . Our aim is to find some new solutions expressed by the Jacobi elliptic function making use of improved F-expansion method.

The organization of the paper is as follows: in Section 2, a brief description of the improved F-expansion for finding traveling wave solutions of nonlinear equations is given. In Sections 3 and 4, we will study, respectively, the KudryashovSinelshchikov equation with the situation $v=\delta=0$ and 
TABLE 1: Relations between values of $r, p, q$, and corresponding $F(\xi)$ in $F^{\prime 2}=q F^{3}+p F^{2}+r F$.

\begin{tabular}{|c|c|c|c|c|}
\hline Case & $r$ & $p$ & $q$ & $F(\xi)$ \\
\hline 1 & 4 & $-4\left(m^{2}+1\right)$ & $4 m^{2}$ & $\operatorname{sn}^{2}(\xi)$ \\
\hline 2 & $4\left(1-m^{2}\right)$ & $4\left(2 m^{2}-1\right)$ & $-4 m^{2}$ & $\mathrm{cn}^{2}(\xi)$ \\
\hline 3 & $-4\left(1-m^{2}\right)$ & $4\left(2-m^{2}\right)$ & -4 & $\mathrm{dn}^{2}(\xi)$ \\
\hline 4 & $m^{2}$ & $2\left(1+m^{2}\right)$ & $m^{2}$ & $(\operatorname{sn}(\xi) \pm i \mathrm{cn}(\xi))^{2}$ \\
\hline 5 & $-\left(1-m^{2}\right)^{2}$ & $2\left(m^{2}-2\right)$ & -1 & $(m \mathrm{cn}(\xi) \pm \operatorname{dn}(\xi))^{2}$ \\
\hline 6 & $1-m^{2}$ & $2\left(1+m^{2}\right)$ & $1-m^{2}$ & $\left(\frac{\operatorname{cn}(\xi)}{1+\operatorname{sn}(\xi)}\right)^{2}$ \\
\hline 7 & $m^{2}-1$ & $2\left(1+m^{2}\right)$ & $m^{2}-1$ & $\left(\frac{\operatorname{dn}(\xi)}{1+m \operatorname{sn}(\xi)}\right)^{2}$ \\
\hline 8 & $m^{2}$ & $2\left(m^{2}-1\right)$ & $m^{2}$ & $\left(\frac{m \operatorname{sn}(\xi)}{1+\operatorname{dn}(\xi)}\right)^{2}$ \\
\hline 9 & 1 & $2\left(1-2 m^{2}\right)$ & 1 & $\left(\frac{\operatorname{sn}(\xi)}{1+\operatorname{cn}(\xi)}\right)^{2}$ \\
\hline
\end{tabular}

$\nu \neq 0, \delta \neq 0$ by the improved F-expansion methods. Finally conclusions are given in Section 5.

\section{Description of the Improved Methods}

Based on F-expansion method [11-13], the main procedures of the improved F-expansion method are as follows.

Step 1. Consider a general nonlinear PDE in the form

$$
F\left(u, u_{x}, u_{t}, u_{x x}, u_{x t}, \ldots\right)=0 .
$$

Using $u(x, t)=U(\xi), \xi=x-c t$, we can rewrite (2) as the following nonlinear ODE:

$$
F\left(U, U^{\prime}, U^{\prime \prime}, \ldots\right)=0
$$

where the prime denotes differentiation with respect to $\xi$.

Step 2. Suppose that the solution of ODE (3) can be written as follows:

$$
U(\xi)=A_{0}+\sum_{i=1}^{n}\left(A_{i} F^{i}(\xi)+B_{i} F^{-i}(\xi)\right)
$$

or

$$
U(\xi)=A_{0}+\sum_{i=1}^{n}\left(A_{i} F^{i}(\xi)+B_{i} F^{i-1} F^{\prime}(\xi)\right),
$$

where $A_{i}, B_{i}(i=1,2, \ldots, n)$ are constants to be determined later and $n$ is a positive integer that is given by the homogeneous balance principle. And $F(\xi)$ satisfies the following equation

$$
\left(F^{\prime}(\xi)\right)^{2}=q F^{3}(\xi)+p F^{2}(\xi)+r F(\xi)
$$

where $r, p$, and $q$ are constant.

Step 3. Substituting (4) or (5) along with (6) into (3) and then setting all the coefficients of $F^{j}(\xi) F^{\prime k}(\xi)(j=1,2, \ldots, k=$ $0,1)$ of the resulting system to zero yield a set of overdetermined nonlinear algebraic equations for $A_{0}, A_{i}$, and $B_{i}(i=$ $1,2, \ldots n)$.
Step 4. Assuming that the constants $A_{0}, A_{i}$, and $B_{i}(i=$ $1,2, \ldots n)$ can be obtained by solving the algebraic equations in Step 3, then by substituting these constants and the solutions of (6) that can be found in Table 1 into (4), we can obtain the explicit solutions of (2) immediately.

\section{Exact Solutions of \\ the Kudryashov-Sinelshchikov Equation in the Case of $\delta=\nu=0$}

Using scale transformation

$$
x=x^{\prime}, \quad t=t^{\prime}, \quad u=\frac{u^{\prime}}{\varepsilon},
$$

we can write the Kudryashov-Sinelshchikov equation (1) in the form

$$
\begin{aligned}
u_{t}+\alpha u u_{x}+u_{x x x} & \\
& -\left(u u_{x x}\right)_{x}-\beta u_{x} u_{x x}-v u_{x x}-\mu\left(u u_{x}\right)_{x}=0,
\end{aligned}
$$

where $\alpha=\gamma / \varepsilon, \beta=\kappa / \varepsilon$, and $\mu=\delta / \varepsilon$ (primes are omitted). When $\delta=\nu=0$, (8) becomes

$$
u_{t}+\alpha u u_{x}+u_{x x x}-\left(u u_{x x}\right)_{x}-\beta u_{x} u_{x x}=0 .
$$

Let

$$
u(x, t)=1-\varphi(\xi), \quad \xi=x-c t,
$$

where $c$ is the wave speed. Under this transformation, (9) can be reduced to the following ordinary differential equation (ODE):

$$
c \varphi^{\prime}-\alpha(1-\varphi) \varphi^{\prime}-\varphi^{\prime \prime \prime}+\left((1-\varphi) \varphi^{\prime \prime}\right)^{\prime}-\beta \varphi^{\prime} \varphi^{\prime \prime}=0 .
$$

Integrating (11) once with respect to $\xi$ and setting the constant of integration to $R$, we have

$$
\frac{1}{2} \alpha \varphi^{2}+(c-\alpha) \varphi-\varphi \varphi^{\prime \prime}-\frac{1}{2} \beta\left(\varphi^{\prime}\right)^{2}+R=0 .
$$


Balancing $\varphi \varphi^{\prime \prime}$ with $\left(\varphi^{\prime}\right)^{2}$ in (12) we find that $n+n+2=$ $2(n+1)$, so $n$ is an arbitrary positive integer. For simplicity, we take $n=2$. Suppose that (12) owns the solutions in the form

$$
\varphi(\xi)=A_{0}+A_{1} F(\xi)+A_{2} F^{2}(\xi)+\frac{B_{1}}{F(\xi)}+\frac{B_{2}}{F^{2}(\xi)} .
$$

Substituting (13) and (6) into (12) and then setting all the coefficients of $F^{k}(k=-5, \ldots, 5)$ of the resulting system to zero, we can obtain the following results:

$$
\begin{aligned}
& A_{1}=A_{2}=B_{2}=0, \\
& c=\frac{2 A_{0} B_{1} p-B_{1}^{2} q+3 r A_{0}-B_{1} p-3 r A_{0}^{2}}{B_{1}}, \\
& \alpha=\frac{3 r A_{0}-B_{1} p}{B_{1}}, \quad \beta=-3, \\
& A_{2}=B_{1}=B_{2}=0 \text {, } \\
& c=\frac{2 A_{0} A_{1} p-A_{1} p+3 q A_{0}-A_{1}^{2} r-3 q A_{0}^{2}}{A_{1}}, \\
& \alpha=\frac{3 q A_{0}-A_{1} p}{A_{1}}, \quad \beta=-3, \\
& A_{2}=0, \quad B_{1}=\frac{A_{1} r}{q}, \quad B_{2}=0, \\
& c=\frac{4 A_{1}^{2} r+2 A_{0} A_{1} p-A_{1} p+3 q A_{0}-3 q A_{0}^{2}}{A_{1}}, \\
& \alpha=\frac{3 q A_{0}-A_{1} p}{A_{1}}, \quad \beta=-3, \\
& A_{0}=\frac{A_{2}(2 w p-r)}{3 q}, \quad A_{1}=2 w A_{2}, \quad B_{1}=B_{2}=0 \text {, } \\
& c=-\frac{-18 q^{2} w+2 A_{2} p^{2} w-6 w A_{2} r q+6 p q-A_{2} p r}{3 q}, \\
& \alpha=-2 p+6 w q, \quad \beta=-\frac{5}{2},
\end{aligned}
$$

where $w=\left(p \pm \sqrt{p^{2}-3 r q}\right) / 3 q, q \neq 0$. Consider the following:

$$
\begin{gathered}
A_{0}=\frac{2 A_{2}(2 w p+5 r)}{3 q}, \quad A_{1}=4 A_{2} w, \\
B_{1}=\frac{4 A_{2} w r}{q}, \quad B_{2}=\frac{A_{2} r^{2}}{q^{2}}, \\
c=-\left(2 \left(-18 w q^{2}+2 A_{2} p^{2} w+24 A_{2} w r q\right.\right. \\
\left.\left.+3 p q-16 A_{2} p r\right)\right) \times(3 q)^{-1}, \\
\alpha=-2 p+12 w q, \quad \beta=-\frac{5}{2},
\end{gathered}
$$

where $w=\left(p \pm \sqrt{p^{2}-3 r q}\right) / 3 q, q \neq 0$

$$
\begin{gathered}
A_{0}=\frac{B_{2}\left(2 w_{1} p-q\right)}{3 r}, \\
A_{1}=A_{2}=0, \quad B_{1}=2 w_{1} B_{2}, \\
c=-\frac{-18 w_{1} r^{2}+2 B_{2} p^{2} w_{1}-6 w_{1} B_{2} q r+6 p r-B_{2} p q}{3 r}, \\
\alpha=-2 p+6 w_{1} r, \quad \beta=-\frac{5}{2},
\end{gathered}
$$

where $w_{1}=\left(p \pm \sqrt{p^{2}-3 r q}\right) / 3 r, r \neq 0$.

Substituting (14)-(19) into (13) with (10), we obtain, respectively, the following formal solution of (9):

$$
u(x, t)=1-A_{0}-\frac{B_{1}}{F(\xi)},
$$

where $\xi=x-\left(\left(2 A_{0} B_{1} p-B_{1}^{2} q+3 r A_{0}-B_{1} p-3 r A_{0}^{2}\right) / B_{1}\right) t$, $\alpha=\left(3 r A_{0}-B_{1} p\right) / B_{1}$, and $\beta=-3$. Consider the following:

$$
u(x, t)=1-A_{0}-A_{1} F(\xi),
$$

where $\xi=x-\left(\left(2 A_{0} A_{1} p-A_{1} p+3 q A_{0}-A_{1}^{2} r-3 q A_{0}^{2}\right) / A_{1}\right) t$, $\alpha=\left(3 q A_{0}-A_{1} p\right) / A_{1}$, and $\beta=-3$. Consider the following:

$$
u(x, t)=1-A_{0}-A_{1} F(\xi)-\frac{A_{1} r}{q F(\xi)},
$$

where $\xi=x-\left(\left(4 A_{1}^{2} r+2 A_{0} A_{1} p-A_{1} p+3 q A_{0}-3 q A_{0}^{2}\right) / A_{1}\right) t$, $\alpha=\left(3 q A_{0}-A_{1} p\right) / A_{1}$, and $\beta=-3$. Consider the following:

$$
u(x, t)=1-\frac{A_{2}(2 w p-r)}{3 q}-2 w A_{2} F(\xi)-A_{2} F^{2}(\xi),
$$

where $\xi=x+\left(\left(-18 q^{2} w+2 A_{2} p^{2} w-6 w A_{2} r q+6 p q-\right.\right.$ $\left.\left.A_{2} p r\right) / 3 q\right) t, \alpha=-2 p+6 w q$, and $\beta=-5 / 2$. Consider the following:

$$
\begin{aligned}
u(x, t)= & 1-\frac{2 A_{2}(2 w p+5 r)}{3 q}-4 w A_{2} F(\xi) \\
& -A_{2}(F(\xi))^{2}-\frac{4 w A_{2} r}{q F(\xi)}-\frac{A_{2} r^{2}}{q^{2} F^{2}(\xi)},
\end{aligned}
$$

where $\xi=x+\left(2\left(-18 w q^{2}+2 A_{2} p^{2} w+24 A_{2} w r q+3 p q-\right.\right.$ $\left.\left.16 A_{2} p r\right) / 3 q\right) t, \alpha=-2 p+12 w q$, and $\beta=-5 / 2$. Consider the following:

$$
u(x, t)=1-\frac{B_{2} w_{1}}{3 r}-\frac{2 B_{2} w_{1}}{F(\xi)}-\frac{B_{2}}{F^{2}(\xi)},
$$

where $\xi=x+\left(\left(-18 w_{1} r^{2}+2 B_{2} p^{2} w_{1}-6 w_{1} B_{2} q r+6 p r-\right.\right.$ $\left.\left.B_{2} p q\right) / 3 r\right) t, \alpha=-2 p+6 w_{1} r$, and $\beta=-5 / 2$.

Combining (20)-(25) with Table 1, many exact solutions of (9) can be obtained. For simplicity, we just give out case 1 of Table 1, the other cases can be discussed similarly. 
When $r=4, p=-4\left(m^{2}+1\right)$, and $q=4 m^{2}$, the solution of elliptic Equation (6) is $F(\xi)=\operatorname{sn}^{2}(\xi, m)$. Substituting it into (20)-(24), we can obtain the following solutions of (9).

From (20), one has

$$
u_{1}(x, t)=1-A_{0}-B_{1} \mathrm{~ns}^{2}(\xi, m),
$$

where $\xi=x+\left(4\left(2 A_{0} B_{1} m^{2}+2 A_{0} B_{1}+B_{1}^{2} m^{2}-3 A_{0}-B_{1} m^{2}-\right.\right.$ $\left.\left.B_{1}+3 A_{0}^{2}\right) / B_{1}\right) t, \alpha=\left(4\left(3 A_{0}+B_{1} m^{2}+B_{1}\right) / B_{1}\right)$, and $\beta=-3$.

When $m \rightarrow 1$ and $\operatorname{sn}(\xi, m) \rightarrow \tanh (\xi)$, (26) becomes

$$
u(x, t)=1-A_{0}-B_{1} \operatorname{coth}^{2}(\xi),
$$

where $\xi=x+\left(4\left(4 A_{0} B_{1}-3 A_{0}-2 B_{1}+B_{1}^{2}+3 A_{0}^{2}\right) / B_{1}\right) t, \alpha=$ $4\left(3 A_{0}+2 B_{1}\right) / B_{1}$, and $\beta=-3$.

When $m \rightarrow 0$ and $\operatorname{sn}(\xi, m) \rightarrow \sin (\xi),(26)$ becomes

$$
u(x, t)=1-A_{0}-B_{1} \sec ^{2}(\xi),
$$

where $\xi=x+\left(4\left(2 A_{0} B_{1}-3 A_{0}-B_{1}+3 A_{0}^{2}\right) / B_{1}\right) t, \alpha=\left(4\left(3 A_{0}+\right.\right.$ $\left.\left.B_{1}\right) / B_{1}\right)$, and $\beta=-3$.

From (21), we have

$$
u(x, t)=1-A_{0}-A_{1} \operatorname{sn}^{2}(\xi, m),
$$

where $\xi=x+4\left(\left(2 A_{0} A_{1} m^{2}+2 A_{0} A_{1}+3 m^{2} A_{0}^{2}+A_{1}^{2}-A_{1} m^{2}-\right.\right.$ $\left.\left.A_{1}-3 m^{2} A_{0}\right) / A_{1}\right) t, \alpha=4\left(\left(A_{1} m^{2}+A_{1}+3 m^{2} A_{0}\right) / A_{1}\right)$, and $\beta=-3$.

When $m \rightarrow 1$, (29) becomes a hyperbolic function solution,

$$
u(x, t)=1-A_{0}-B_{1} \tanh ^{2}(\xi),
$$

where $\xi=x+\left(4\left(4 A_{0} B_{1}-3 A_{0}-2 B_{1}+B_{1}^{2}+3 A_{0}^{2}\right) / B_{1}\right) t, \alpha=$ $\left(4\left(3 A_{0}+2 B_{1}\right) / B_{1}\right)$, and $\beta=-3$.

When $m \rightarrow 0$, (29) becomes a trigonometric function solution,

$$
u(x, t)=1-A_{0}-B_{1} \sin ^{2}(\xi),
$$

where $\xi=x+4\left(2 A_{0}+A_{1}-1\right) t, \alpha=4$, and $\beta=-3$.

From (22), we have

$$
u(x, t)=1-A_{0}-A_{1} \operatorname{sn}^{2}(\xi, m)-\frac{A_{1} r}{q} \mathrm{~ns}^{2}(\xi, m),
$$

where $\xi=x+\left(4\left(2 A_{0} A_{1} m^{2}+2 A_{0} A_{1}+3 m^{2} A_{0}^{2}-4 A_{1}^{2}-A_{1} m^{2}-\right.\right.$ $\left.\left.A_{1}-3 m^{2} A_{0}\right) / A_{1}\right) t, \alpha=\left(4\left(A_{1} m^{2}+A_{1}+3 m^{2} A_{0}\right) / A_{1}\right)$, and $\beta=-3$.

When $m \rightarrow 1$, (32) becomes a hyperbolic function solution,

$$
u(x, t)=1-A_{0}-A_{1} \tanh ^{2}(\xi)-\frac{A_{1} r}{q} \operatorname{coth}^{2}(\xi),
$$

where $\xi=x+\left(4\left(4 A_{0} A_{1}+3 A_{0}^{2}-4 A_{1}^{2}-2 A_{1}-3 A_{0}\right) / A_{1}\right) t$, $\alpha=\left(4\left(2 A_{1}+3 A_{0}\right) / A_{1}\right)$, and $\beta=-3$.

From (23), we have

$$
\begin{aligned}
u(x, t)= & 1+\frac{A_{2}\left(2 w\left(m^{2}+1\right)+1\right)}{3 m^{2}} \\
& -2 w A_{2} \operatorname{sn}^{2}(\xi, m)-A_{2} \operatorname{sn}^{4}(\xi, m),
\end{aligned}
$$

where $w=\left(-m^{2}-1 \pm \sqrt{m^{4}-m^{2}+1}\right) / 3 m^{2}, \xi=x+$ $\left(4\left(-2 w A_{2} m^{2}+2 w A_{2} m^{4}+2 w A_{2}-18 w m^{4}+A_{2} m^{2}+A_{2}-\right.\right.$ $\left.\left.6 m^{4}-6 m^{2}\right) / 3 m^{2}\right) t, \alpha=8\left(3 w m^{2}+m^{2}+1\right)$, and $\beta=-5 / 2$.

When $m \rightarrow 1$, (34) becomes a hyperbolic function solution,

$$
u(x, t)=1-\frac{1}{12} A_{2} w-2 w A_{2} \tanh ^{2}(\xi)-A_{2} \tanh ^{4}(\xi, x),
$$

where $w=(-2 \pm 1) / 3, \xi=8\left(3 w-(1 / 3) A_{2} w+2-(1 / 3) A_{1}\right) t$, $\alpha=16+24 w$, and $\beta=-5 / 2$.

From (24), we have

$$
\begin{aligned}
u(x, t)= & 1+\frac{2 A_{2}\left(2 w\left(m^{2}+1\right)-5\right)}{3 m^{2}} \\
& -4 w A_{2} \operatorname{sn}^{2}(\xi, m)-A_{2} \mathrm{sn}^{4}(\xi, m) \\
& -\frac{4 w A_{2}}{m^{2}} \mathrm{~ns}^{2}(\xi, m)-\frac{A_{2}}{m^{4}} \mathrm{~ns}^{4}(\xi, m),
\end{aligned}
$$

where $w=\left(-m^{2}-1 \pm \sqrt{m^{4}+14 m^{2}+1}\right) / 6 m^{2}, \xi=x+$ $\left(8\left(-18 w m^{4}+2 w A_{2} m^{4}+28 w A_{2} m^{2}+2 w A_{2}-3 m^{4}-3 m^{2}+\right.\right.$ $\left.\left.16 A_{2} m^{2}+16 A_{2}\right) / 3 m^{2}\right) t, \alpha=8\left(m^{2}+1+6 w m^{2}\right)$, and $\beta=-5 / 2$.

When $m \rightarrow 1$, (36) becomes a hyperbolic function solution,

$$
\begin{aligned}
u(x, t)= & 1+\frac{2}{3} A_{2}(4 w-5)-4 w A_{2} \tanh ^{2}(\xi) \\
& -A_{2} \tanh ^{4}(\xi)-4 w A_{2} \operatorname{coth}^{2}(\xi)-A_{2} \operatorname{coth}^{4}(\xi),
\end{aligned}
$$

where $w=(-1 \pm 2) / 3, \xi=x-\left(48 w-(256 / 3) w A_{2}+16-\right.$ $\left.(256 / 3) A_{2}\right) t, \alpha=16+48 w$, and $\beta=-5 / 2$.

From (25), we have

$$
\begin{aligned}
u(x, t)= & 1+\frac{1}{3} B_{2}\left(2 w\left(m^{2}+1\right)+m^{2}\right) \\
& -2 w B_{2} \mathrm{~ns}^{2}(\xi, m)-B_{2} \mathrm{~ns}^{4}(\xi, m),
\end{aligned}
$$

where $w=\left(-m^{2}-1 \pm \sqrt{m^{4}-m^{2}+1}\right) / 3, \xi=x+$ $\left((8 / 3) w B_{2} m^{4}+(8 / 3) w B_{2} m^{2}-(8 / 3) w B_{2}+24 w+8 m^{2}+8-\right.$ $\left.(4 / 3) B_{2} m^{4}-(4 / 3) B_{2} m^{2}\right) t, \alpha=8\left(3 w+m^{2}+1\right)$, and $\beta=-5 / 2$.

When $m \rightarrow 1$, (38) becomes a hyperbolic function solution,

$$
\begin{aligned}
u(x, t)= & 1+\frac{1}{3} B_{2}(4 w+1) \\
& -2 w B_{2} \operatorname{coth}^{2}(\xi)-B_{2} \operatorname{coth}^{4}(\xi),
\end{aligned}
$$

where $w=(-2 \pm 1) / 3, \xi=x-\left(24 w-(8 / 3) w B_{2}+16-(8 / 3) B_{2}\right) t$, $\alpha=16+24 w$, and $\beta=-5 / 2$.

When $m \rightarrow 0$, (38) becomes a triangle function solution,

$$
u(x, t)=1+\frac{2}{3} w B_{2}-2 w B_{2} \csc ^{2}(\xi)-B_{2} \csc ^{4}(\xi),
$$

where $w=(-1 \pm 1) / 3, \xi=x-\left(24 w+8-(8 / 3) B_{2} w\right) t, \alpha=$ $24 w+8$, and $\beta=-5 / 2$. 
We notice that when $\beta=-3$, some Jacobi elliptic function solutions have been given in [8]. Part of our results may be the same with them. However, when $\beta=-(5 / 2)$, the Jacobi elliptic function solutions of (9) have not been reported in the related literatures, so we believe that our solutions (34), (36), and (38) are new.

\section{Exact Solutions of}

\section{the Kudryashov-Sinelshchikov Equation in the Case of $\delta \neq 0, \mu \neq 0$}

In this case, by similar process, (1) can be changed into the following PDE:

$$
\begin{aligned}
\frac{1}{2} \alpha \varphi^{2} & +(c-\alpha) \varphi-\varphi \varphi^{\prime \prime} \\
& -\frac{1}{2} \beta\left(\varphi^{\prime}\right)^{2}+\nu \varphi^{\prime}+\mu(1-\varphi) \varphi^{\prime}+R=0,
\end{aligned}
$$

where $\varphi^{\prime}=d \varphi / d \xi, \xi=x-c t$, and $R$ is an integral constant.

Suppose that (41) owns the solutions in the form

$$
\begin{aligned}
\varphi(\xi)= & A_{0}+A_{1} F(\xi)+A_{2} F^{2}(\xi) \\
& +B_{1} F(\xi)^{\prime}+B_{2} F(\xi) F(\xi)^{\prime} .
\end{aligned}
$$

Substituting (42) and (6) into (41) and then setting all the coefficients of $F^{j}(\xi) F^{\prime k}(\xi)(j=0,1, \ldots, 6, k=0,1)$ of the resulting system to zero, we can obtain the following results:

$$
\begin{gathered}
A_{0}=0, \quad A_{1}=w B_{1}, \quad A_{2}=B_{2}=0, \\
c=-\frac{5}{3} w B_{1} r-\frac{4}{3} p, \quad \alpha=-\frac{4}{3} p, \\
\beta=-\frac{8}{3}, \quad \lambda=\frac{2}{3} B_{1} r+\frac{1}{3} w, \quad \mu=-\frac{1}{3} w,
\end{gathered}
$$

where $w= \pm \sqrt{p}$. Consider the following:

$$
\begin{gathered}
A_{0}=\frac{B_{2} w\left(2 p w^{2}-2 p^{2}+3 r q\right)}{9 q^{2}}, \\
A_{1}=\frac{2 B_{2} w(w-p)}{3 q}, \\
A_{2}=-B_{2} w, \quad B_{1}=-\frac{B_{2}\left(w^{2}-p\right)}{3 q}, \\
c=-w\left(14 w^{2} B_{2} p^{2}-42 w^{2} B_{2} r q+63 B_{2} r p q\right. \\
\left.+108 w q^{2}-14 B_{2} p^{3}\right) \times\left(45 q^{2}\right)^{-1}, \\
\alpha=-\frac{12}{5} w^{2}, \quad \beta=-\frac{12}{5}, \\
\lambda=-\left(2 w^{2} B_{2} p^{2}-2 B_{2} p^{3}+9 B_{2} r p q\right. \\
\left.-6 w^{2} B_{2} r q+9 w q^{2}\right) \times\left(45 q^{2}\right)^{-1}, \\
\mu=\frac{1}{5} w
\end{gathered}
$$

where $w= \pm \sqrt[4]{p^{2}-3 r q}$.
Substituting (43) and (44) along with (10) into (42), we obtain, respectively, the following formal solution of (8):

$$
u(x, t)=1-w B_{1} F(\xi)-B_{1} F^{\prime}(\xi),
$$

where $w= \pm \sqrt{p}, \xi=x+\left((5 / 3) w B_{1} r+(4 / 3) p\right) t, \alpha=$ $-(4 / 3) p, \beta=-8 / 3, \lambda=(2 / 3) B_{1} r+(1 / 3) w$, and $\mu=-(1 / 3) w$. Consider the following:

$$
\begin{aligned}
u(x, t)= & 1-\frac{B_{2} w\left(2 p w^{2}-2 p^{2}+3 r q\right)}{9 q^{2}} \\
& -\frac{2 B_{2} w\left(w^{2}-p\right) F(\xi)}{3 q}+w B_{2} F^{2}(\xi) \\
& +\frac{B_{2}\left(w^{2}-p\right) F^{\prime}(\xi)}{3 q}-B_{2} F(\xi) F^{\prime}(\xi),
\end{aligned}
$$

where $w= \pm \sqrt[4]{p^{2}-3 r q}, \xi=x+\left(w\left(14 w^{2} B_{2} p^{2}-42 w^{2} B_{2} r q+\right.\right.$ $\left.\left.63 B_{2} r p q+108 w q^{2}-14 B_{2} p^{3}\right) / 45 q^{2}\right) t, \alpha=-(12 / 5) w^{2}, \beta=$ $-12 / 5, \lambda=-\left(\left(2 w^{2} B_{2} p^{2}-2 B_{2} p^{3}+9 B_{2} r p q-6 w^{2} B_{2} r q+\right.\right.$ $\left.\left.9 w q^{2}\right) / 45 q^{2}\right)$, and $\mu=(1 / 5) w$.

Combining (45) and (46) with Table 1, many exact solutions of (8) can be obtained. For simplicity, we just give out case 3 of Table 1 , and the other cases can be discussed similarly.

When $r=-4\left(1-m^{2}\right), p=4\left(2-m^{2}\right), q=-4$, and the solution of elliptic Equation (6) is $F(\xi)=\operatorname{dn}^{2}(\xi, m)$. Substituting it into (45) and (46), we can obtain the following Jacobi elliptic function solutions of (8):

$$
\begin{aligned}
u(x, t)=1-B_{1}( & w \operatorname{dn}^{2}(\xi, m) \\
& \left.-2 m^{2} \operatorname{sn}(\xi, m) \operatorname{cn}(\xi, m) \operatorname{dn}(\xi, m)\right),
\end{aligned}
$$

where $w= \pm 2 \sqrt{2-m^{2}}, \xi=x-\left((20 / 3) w B_{1}-(20 / 3) w B_{1} m^{2}-\right.$ $\left.(32 / 3)+(16 / 3) m^{2}\right) t, \alpha=(16 / 3)\left(m^{2}-2\right), \beta=-8 / 3, v=$ $(1 / 3)\left(-8 B_{1}+8 B_{1} m^{2}+w\right), \mu=-(1 / 3) w$, and

$$
\begin{aligned}
u(x, t)= & 1-\frac{B_{2} w\left(2 p w^{2}-2 p^{2}+3 r q\right)}{9 q^{2}} \\
& -\frac{2 B_{2} w\left(w^{2}-p\right) \operatorname{dn}^{2}(\xi, m)}{3 q}+w B_{2} \operatorname{dn}^{4}(\xi, m) \\
& -\frac{2 m^{2} B_{2}\left(w^{2}-p\right) \operatorname{dn}(\xi, m) \operatorname{cn}(\xi, m) \operatorname{sn}(\xi, m)}{3 q}
\end{aligned}
$$$$
+2 m^{2} B_{2} \operatorname{dn}^{3}(\xi, m) \mathrm{cn}(\xi, m) \operatorname{sn}(\xi, m),
$$

where $w= \pm 2 \sqrt[4]{1-m^{2}-m^{4}}, \xi=x+(2 w / 45)\left(7 w^{2} B_{2}-\right.$ $7 w^{2} B_{2} m^{2}+28 B_{2}-42 B_{2} m^{2}-42 B_{2} m^{4}+7 w^{2} B_{2} m^{4}+28 B_{2} m^{6}+$ $54 w) t, \alpha=-(12 / 5) w^{2}, \beta=-(12 / 5), v=-(2 / 45) w^{2} B_{2}+$ $(2 / 45) w^{2} B_{2} m^{2}-(2 / 45) w^{2} B_{2} m^{4}-(8 / 45) B_{2}+(4 / 15) B_{2} m^{2}+$ $(4 / 15) B_{2} m^{4}-(8 / 45) B_{2} m^{6}-(1 / 5) w$, and $\mu=(1 / 5) w$. 
Similarly, when $m \rightarrow 1$ we can obtain hyperbolic function solutions of (8). Here, we omit them.

We indicate that these results with $\beta=-12 / 5$ and $\beta=$ $-8 / 3$ are new.

\section{Conclusions}

The Jacobi elliptic function solutions of (1) are only reported in [8] with special case of $\beta=-3$ and $\beta=-4$. In the present work, we successfully obtained some new Jacobi elliptic function solutions of the Kudryashov-Sinelshchikov equation with $\beta=-3, \beta=-5 / 2, \beta=-8 / 3$, and $\beta=$ $-12 / 5$ using the improved F-expansion method. When the modulus $\mathrm{m}$ of the Jacobi elliptic function is driven to the limits 1 and 0 , some exact solutions expressed by hyperbolic function and trigonometric function can also be obtained. All the results we obtained have been verified. The related results are enriched.

\section{Acknowledgments}

This research is supported by the Natural Science Foundation of China (11161020), the Natural Science Foundation of Yunnan (2013FZ117), and Research Foundation of Honghe University (10XJY120).

\section{References}

[1] N. A. Kudryashov and D. I. Sinelshchikov, "Nonlinear waves in bubbly liquids with consideration for viscosity and heat transfer," Physics Letters A, vol. 374, no. 19-20, pp. 2011-2016, 2010.

[2] P. N. Ryabov, "Exact solutions of the Kudryashov-Sinelshchikov equation," Applied Mathematics and Computation, vol. 217, no. 7, pp. 3585-3590, 2010.

[3] M. Randrüüt, "On the Kudryashov-Sinelshchikov equation for waves in bubbly liquids," Physics Letters A, vol. 375, pp. 36873692, 2011.

[4] J. Li, "Exact travelling wave solutions and their bifurcations for Kudryashov-Sinelshchikovequation," International Journal of Bifurcation and Chaos, vol. 5, Article ID 125011, 19 pages, 2012.

[5] B. He, Q. Meng, and Y. Long, "The bifurcation and exact peakons, solitary and periodic wave solutions for the Kudryashov-Sinelshchikov equation," Communications in Nonlinear Science and Numerical Simulation, vol. 17, no. 11, pp. 4137-4148, 2012.

[6] B. He, Q. Meng, J. Zhang, and Y. Long, "Periodic loop solutions and their limit forms for the Kudryashov-Sinelshchikov equation," Mathematical Problems in Engineering, vol. 2012, Article ID 320163, 10 pages, 2012.

[7] M. Nadjafikhah and V. Shirvani-Sh, "Lie symmetry analysis of Kudryashov-Sinelshchikov equation," Mathematical Problems in Engineering, vol. 2011, Article ID 457697, 9 pages, 2011.

[8] Y. He, "Exact solutions of the Kudryashov-Sinelshchikov equation using the multiple $G^{\prime} / G$-expansion method," Mathematical Problems in Engineering, vol. 2013, Article ID 708049, 7 pages, 2013.

[9] O. Y. Efimova, "The modified simplest equation method to look for exact solutions of nonlinear partial differential equations," http://arxiv.org/abs/1011.4606, 2010.
[10] M. Mirzazadeh and M. Eslami, "Exact solutions of the Kudryashov-Sinelshchikov equation and nonlinear telegraph equation via the first integral method," Nonlinear Analysis. Modelling and Control, vol. 17, no. 4, pp. 481-488, 2012.

[11] N. A. Kudryashov, "Exact solutions of the generalized Kuramoto-Sivashinsky equation," Physics Letters A, vol. 147, no. 5-6, pp. 287-291, 1990.

[12] N. A. Kudryashov, "Simplest equation method to look for exact solutions of nonlinear differential equations," Chaos, Solitons and Fractals, vol. 24, no. 5, pp. 1217-1231, 2005.

[13] M. Wang and X. Li, "Applications of F-expansion to periodic wave solutions for a new Hamiltonian amplitude equation," Chaos, Solitons and Fractals, vol. 24, no. 5, pp. 1257-1268, 2005. 


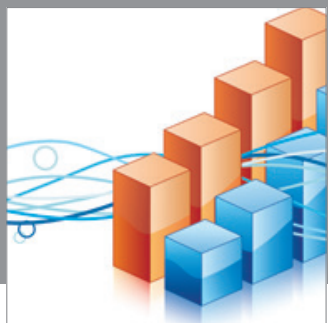

Advances in

Operations Research

mansans

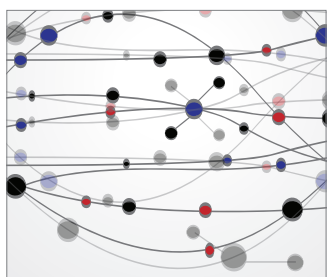

The Scientific World Journal
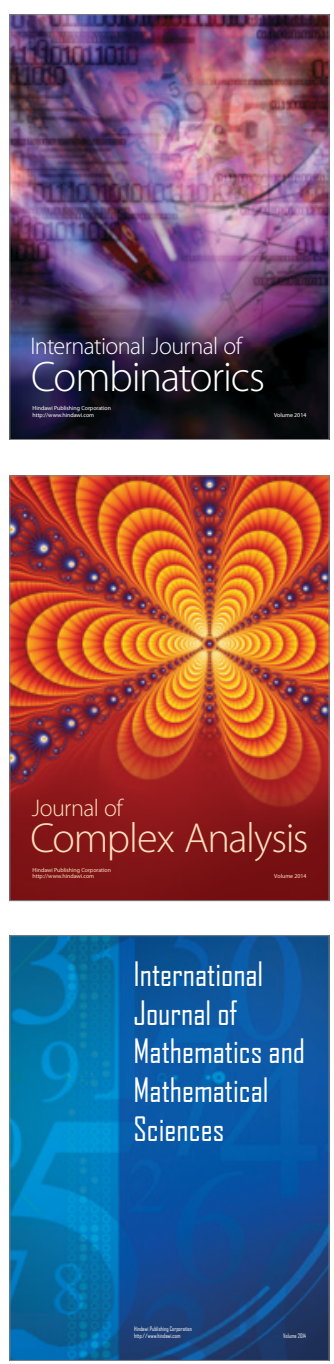
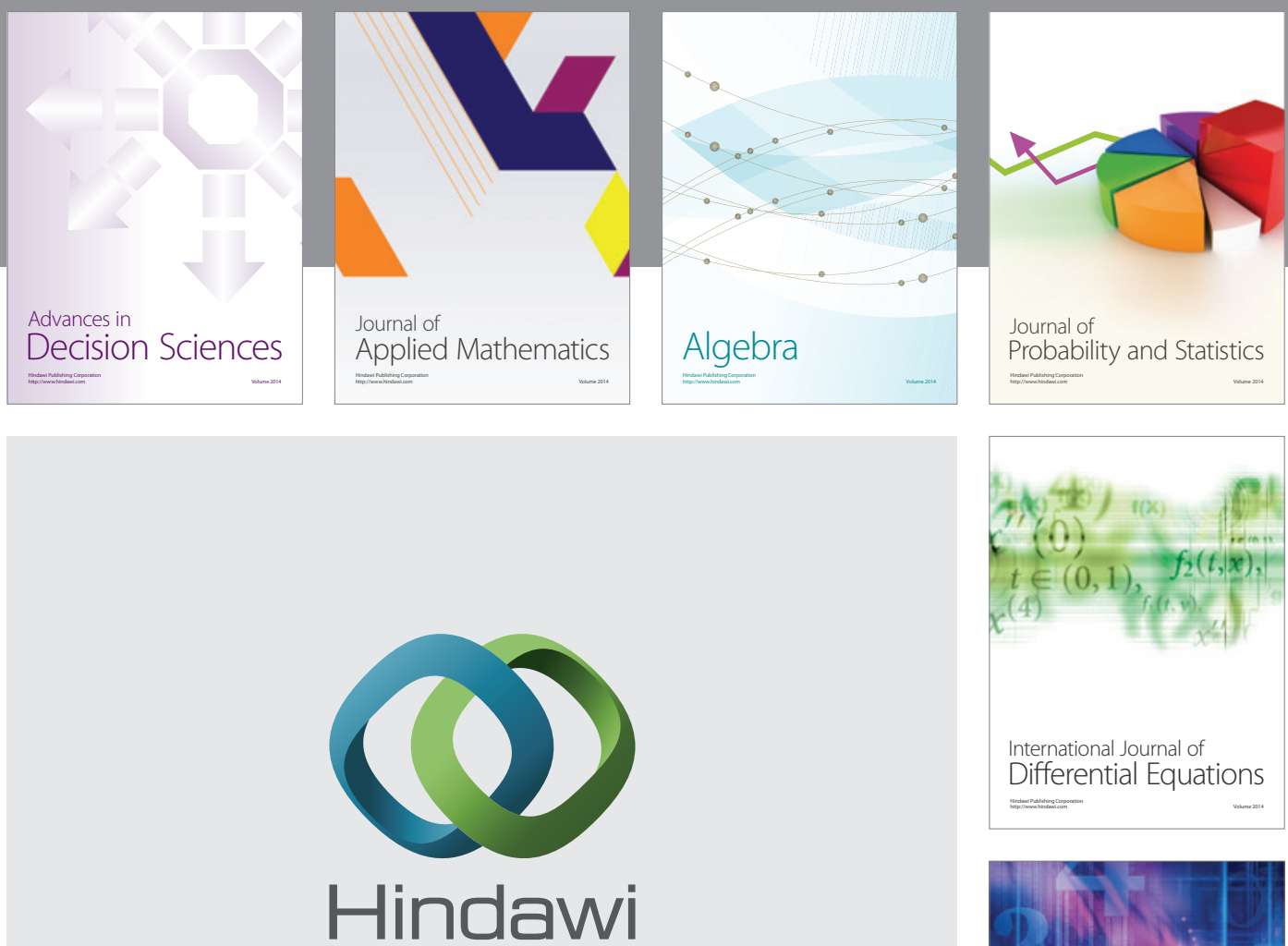

Submit your manuscripts at http://www.hindawi.com
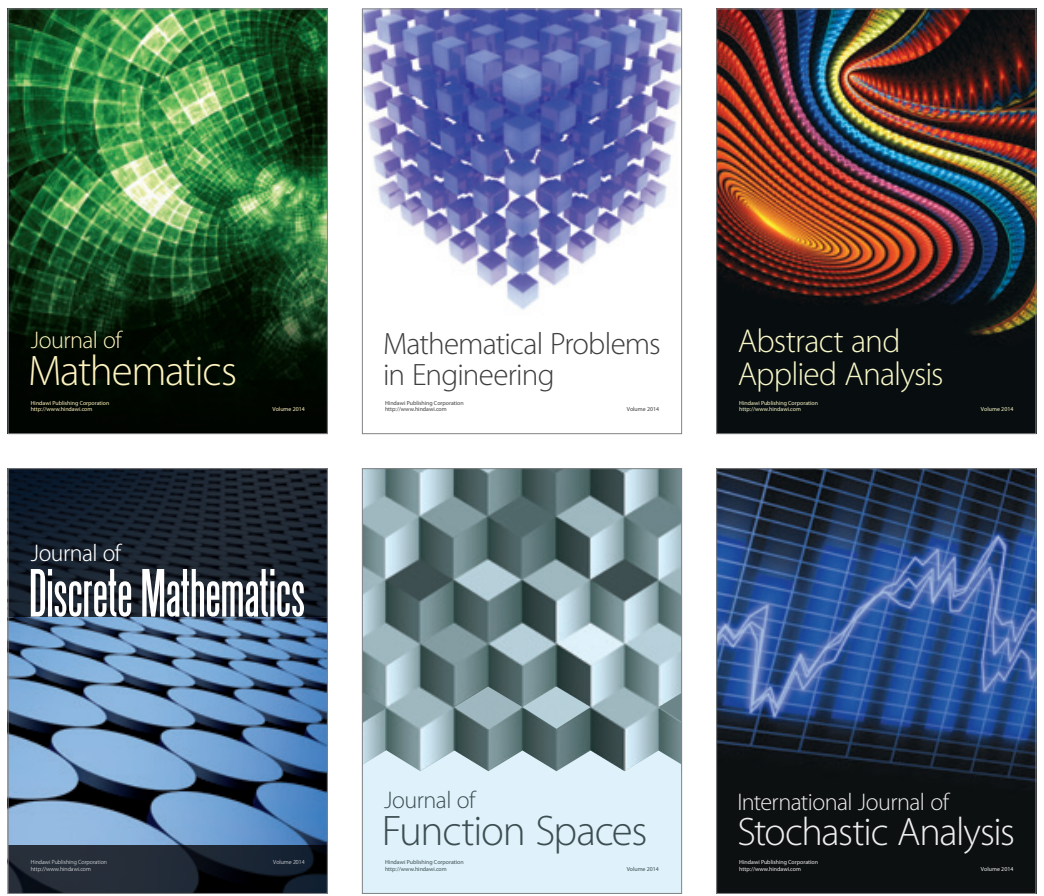

Journal of

Function Spaces

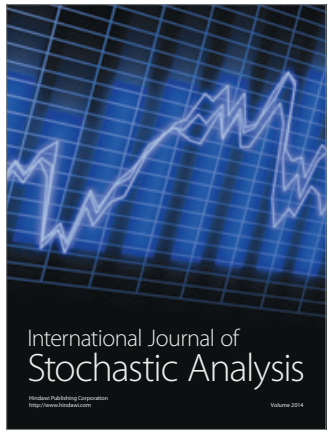

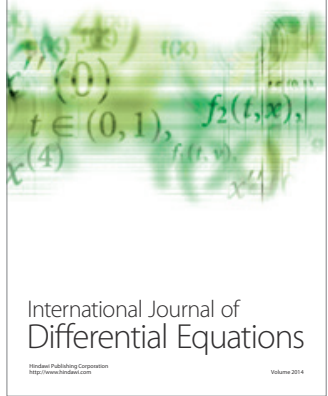
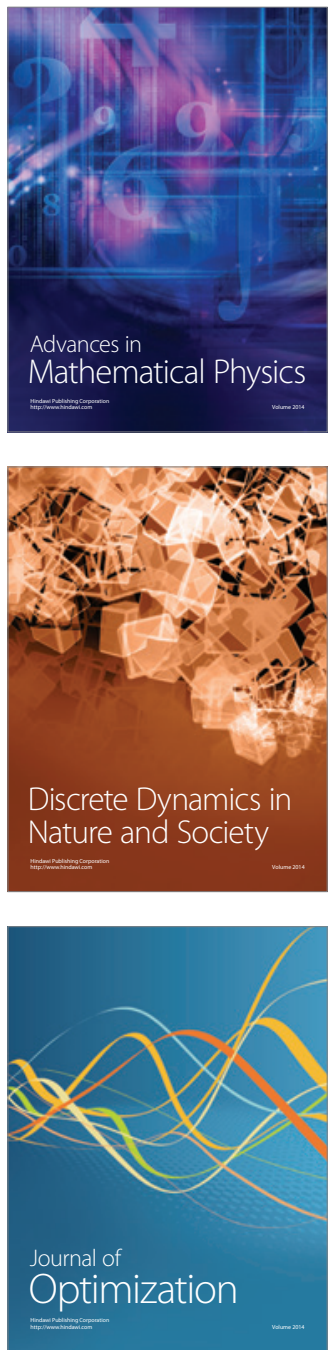